\title{
Implementación y evaluación de la eficacia de un Programa de Educación Emocional en Adolescentes con diagnóstico de TDAH
}

\section{Implementation and evaluation of the efficiency of an Emotional Education Program in Teenagers diagnosed with ADHD}

\author{
Alicia Pérez de Albéniz Iturriaga, Eduardo Fonseca Pedrero \\ Universidad de la Rioja, España \\ Raquel Carballido Guisado, Víctor Santiago Sigüenza Marín \\ Comunidad Autónoma de La Rioja, España. \\ Beatriz Lucas Molina \\ Universidad de Valencia, España.
}

(Rec: enero de 2019 - Acept: octubre de 2019)

\section{Resumen}

Las habilidades socioemocionales en jóvenes con diagnóstico de Trastorno por Déficit de Atención e Hiperactividad (TDAH) son esenciales en su desarrollo psicológico y bienestar personal. Sin embargo, a pesar del reconocimiento general de la necesidad de dotar a estos jóvenes de recursos y estrategias que no están ni atendidas ni contempladas en el currículo académico, son escasos los trabajos que muestren la validez de programas de educación emocional en este colectivo. El objetivo del trabajo fue triple: a) la adaptación e implementación de un programa de educación emocional (denominado INTEMO), b) la evaluación de su eficacia, y c) el análisis de la satisfacción con el programa de usuarios y familiares. Se utilizó un grupo de tratamiento $(n=24)$ y un grupo de comparación ( $n=24)$ de estudiantes con diagnóstico de TDAH, que fueron evaluados en dos momentos temporales. Los resultados evidenciaron que los participantes que recibieron el programa mejoraron de forma estadísticamente significativa en la dimensión de problemas con los compañeros y en regulación emocional, respecto al grupo de comparación. No se observaron diferencias estadísticamente significativas entre los grupos en autoestima y empatía. Los usuarios del programa indicaron una alta satisfacción con la intervención. Los resultados obtenidos indican la necesidad de continuar con el análisis de la eficacia de este programa y se discute la necesidad de potenciar las competencias socioemocionales en esta etapa, con el fin de mejorar el bienestar personal, familiar, académico y social de la población infantojuvenil con TDAH.

Palabras claves: TDAH, educación emocional, inteligencia emocional, programa INTEMO, autoestima, empatía.

\begin{abstract}
Socio-emotional skills in teenagers diagnosed with Attention-deficit/hyperactivity disorder (ADHD) are essential for their psychological development and subjective well-being. However, despite the general recognition for the need to provide young people diagnosed with ADHD with resources and strategies, that are neither addressed nor contemplated in the academic curriculum, there is a lack of studies that shed a light on the effects of emotional intelligence programs in this group. The objective of this work was threefold: a) the adaptation and implementation of an emotional intelligence program (named INTEMO), b) the evaluation of its effectiveness, and c) the analysis of satisfaction of participants and their families with the program. An experimental group was set up ( $n=24)$ together with a comparison group $(n=24)$ of students diagnosed with ADHD, and both were evaluated at two different points. The results obtained demonstrated that, after three months of intervention, the experimental group showed significantly higher scores with regard to peer problems and emotional regulation, compared to the comparison group. No significant results were found on empathy and self-esteem. The participants of the program expressed high satisfaction with the intervention. The results indicate the need to continue with the analysis of the effectiveness of this program, with the aim of promoting the socio-emotional competences at this stage in order to improve well-being in personal, family, academic and social spheres among the infant-juvenile population with ADHD.
\end{abstract}

Keywords: ADHD, emotional education, emotional intelligence, INTEMO program, self-esteem, empathy. 


\section{Introducción}

El Trastorno por Déficit de Atención e Hiperactividad (TDAH) se podría delimitar, según los sistemas clasificatorios internacionales, como un conjunto de síntomas de hiperactividad, impulsividad y/o inatención que se presentan en varios contextos y que genera un impacto clínicamente significativo a nivel personal, familiar, social y/o académico (American Psychiatric Association [APA], 2014). Es un problema de comportamiento con una prevalencia estimada del 5,9 y el $7,1 \%$ en niños y adolescentes respectivamente (Willcutt, 2012). El TDAH se caracteriza por dificultades para adaptarse a su entorno como, por ejemplo, al ámbito académico (Padrós, Zamora, Salinas y Martínez, 2016), así como por dificultades en el funcionamiento cognitivo (p.ej., funciones ejecutivas, memoria de trabajo) (APA, 2014; Barkley, 2014). Sin embargo, se debe tener especialmente en cuenta que no todos los niños y niñas ni adolescentes con diagnóstico de TDAH experimentan los mismos síntomas ni lo vivencian con la misma intensidad, gravedad y duración (Barkley, 2014; Mena, Nicolau, Salat, Tort y Romero, 2008; Sasser, Kalvin y Bierman, 2016). Existen muchos modelos etiológicos para la explicación del TDAH. En este trabajo, el TDAH se entiende no como una enfermedad cerebral (o biológica), sino más bien como un problema del comportamiento (García de Vinuesa, González Pardo y Pérez-Álvarez, 2014). Se concibe su etiología desde una perspectiva esencialmente bio-psico-social y no bio-bio-bio, donde una amplia amalgama de factores procedentes de múltiples niveles de análisis (que se encuentran en interacción), entra en juego en su explicación y mantenimiento (Pérez-Álvarez, 2018). Desde este acercamiento, se evitan reduccionismos insulsos, concibiendo el TDAH como un sistema complejo dinámico (Fonseca-Pedrero, 2018) y posibilitando una intervención psicológica y educativa donde la implicación y coordinación entre la familia, la escuela y los diferentes profesionales involucrados es nuclear.

Existen ejemplos de intervenciones desde el contexto escolar que se han centrado en la mejora en el proceso de enseñanza-aprendizaje de los jóvenes con diagnóstico de TDAH. Por ejemplo, el programa Challenging Horizons Program ( $\mathrm{CHP}$ ) fue desarrollado por Evans, Axelrod y Langberg (2004) con una muestra de siete adolescentes (12-14 años) y más tarde fue modificado con la finalidad de incorporar estrategias educativas, de habilidades sociales e intervenciones familiares (Evans, Scultz, Demars y Davis, 2011). Los resultados del análisis sobre su eficacia evidenciaron mejoras significativas en relación con el rendimiento académico y presentaron una mejoría en los síntomas de hiperactividad/impulsividad. Asimismo, el programa Summer Treatment Program (Sibley, PeIham, Evans, Gnag, Ross y Greiner, 2011) fue una intervención escolar en adolescentes con TDAH en edades comprendidas entre los 11 y 16 años, que mostró mejoras en relación con los problemas de conducta, inatención, socialización, estados de ánimo y rendimiento académico. Por su parte, el programa Supporting Teens Academic Needs Daily (STAND) (Sibley, PeIham, Derefinko, Kuriyan, Sánchez y Graziano, 2013) se llevó a cabo con adolescentes con TDAH con edades comprendidas entre los 11 y 15 años. El análisis de su impacto indicó que el grupo que acudió a la intervención obtuvo mejor rendimiento académico, un incremento en las habilidades de planificación, una disminución de los problemas escolares y una disminución significativa de síntomas relacionados con este trastorno. Estas intervenciones tenían como foco principal la mejora de los procesos de enseñanza-aprendizaje de adolescentes con diagnóstico de TDAH. Efectivamente, los síntomas de inatención pueden deteriorar su rendimiento académico provocando fracaso escolar (y viceversa), pero existen otros aspectos este problema comportamental que deben ser atendidos como, por ejemplo, la gestión de las emociones, la autorregulación y las relaciones sociales. En concreto, se ha señalado que los síntomas asociados con la impulsividad pueden llegar a causar problemas de comportamiento y problemas en sus relaciones sociales y familiares (Garaigordobil, Cruz y Pérez, 2003; González, Navarro, Grau, Galipienso y Fernández, 2014; López, Sánchez, Pérez-Nieto y Fernández, 2008). Además, niños y jóvenes con hiperactividad son más espontáneos, expresivos e impulsivos y esto puede traducirse en problemas para el manejo adecuado de las emociones (Campeño-Martínez, Gázquez-Linares y Santiuste-Bermejo, 2016). De hecho, la investigación ha indicado que esta población muestra un mayor riesgo de presentar problemas conductuales, emocionales y de autoestima (Evans et al., 2014; Larson, Moneta, Richards y Wilson, 2001; Fernández-Jaén et al., 2011; Wehmeier, Schacht y Barkley, 2010).

Las investigaciones mencionadas ponen de relieve la necesidad de llevar a cabo intervenciones con la finalidad de desarrollar y promocionar las competencias socioemocionales en este colectivo, como pudiera ser la percepción o la regulación emocional de uno mismo o de los otros (Decety y Svetlova, 2012). Por lo tanto, parece sensato diseñar, implementar y evaluar programas de educación emocional con la finalidad de dotar a los jóvenes de recursos y estrategias que no están ni atendidas ni contempladas en el currículo académico, como pudieran ser, las competencias socioemocionales.

El ámbito del desarrollo de competencias socioemocionales de la mano de los programas de Inteligencia Emocional (IE) ofrece una perspectiva en la que el desarrollo y la puesta en práctica de habilidades emocionales se revelan como claves para conseguir el bienestar psicológico y adaptación del individuo al entorno (Bisquerra, 2011; Extremera y Fernández-Berrocal, 2013). En el campo de la intervención en IE en jóvenes destaca el programa INTEMO (Cabello, Castillo, Rueda y Fernández-Berrocal, 2016; Extremera y Fernández-Berrocal, 2013; Ruiz-Aranda et al., 2013). Dirigido a jóvenes de entre 12 y 18 años, se centra en el aprendizaje de capacidades emocionales que componen la IE, esto es, las capacidades de percibir, expresar, facilitar, comprender y regular las emociones propias y las de los demás, las cuales pertenecen a las cuatro ramas del modelo teórico de Mayer y Salovey (1997). Los resultados del examen de la eficacia de este programa indican su efectividad para promover y mejorar la salud mental de los adolescentes (Cabello et al., 2016; Castillo, Salguero, Fernández-Berrocal y Balluerka, 2013; Ruiz-Aranda et al., 2013). Debido a los beneficios que se derivan de la implementación de este programa, no cabe duda de que sería de sumo interés su implementación y validación en alumnos con diagnóstico de TDAH.

Por todo lo señalado, la educación emocional se presenta como una intervención necesaria en los adolescentes con diagnóstico de TDAH. Sin embargo, a pesar del reconocimiento por parte de la comunidad científica y educativa de la necesidad de intervenir psico-educativamente en el alumnado con diagnóstico de TDAH y de la preocupación por parte de las instituciones educativas por desarrollar competencias socioemocionales, son escasos los estudios científicos que analicen 
el impacto de este tipo intervenciones o las evidencias científicas obtenidas son aún débiles (Storebø et al., 2019). Dentro de este contexto de investigación, la finalidad del presente estudio fue triple: a) implementar un programa de educación emocional (denominado INTEMO) en alumnado con diagnóstico de TDAH; b) analizar la eficacia del programa a través de indicadores de salud mental, inteligencia emocional percibida, empatía y autoestima; y c) examinar la satisfacción con el programa tanto de usuarios como de sus familiares (aceptabilidad). Se hipotetiza que el programa INTEMO podrá ser adaptado e implementado en adolescentes con diagnóstico de TDAH. Además, se espera que los participantes del grupo de tratamiento referirán mejorías tras la intervención, respecto al grupo de comparación, en aspectos emocionales y afectivos y, finalmente, que los usuarios del programa referirán una elevada satisfacción hacia el programa de intervención.

\section{Método}

\section{Participantes}

El diseño de investigación fue un diseño longitudinal de tipo cuasiexperimental con evaluación pre y post tratamiento, con un grupo de comparación y un grupo de tratamiento/intervención de alumnos con diagnóstico de TDAH.

Con la ayuda de la asociación regional de padres de hijos hiperactivos (ARPANIH, Asociación Riojana de Padres de Niños Hiperactivos) se recabó una muestra de conveniencia compuesta por un total de 48 alumnos con diagnóstico de TDAH. Se dividió aleatoriamente la muestra en dos grupos: a) grupo de tratamiento que participó en el programa de intervención; b) grupo de comparación que únicamente participó en las sesiones de evaluación pre y postest.

Todos los participantes fueron alumnos de Educación Secundaria Obligatoria (ESO), con edades comprendidas entre los 12 y 15 años con diagnóstico de TDAH. El grupo de intervención lo formaron 24 participantes (de los que 18 fueron chicos) con una media de edad de 13,42 años $(D T=1,2)$. El grupo control lo integraron 24 alumnos (de los que 21 eran chicos) con una media de edad de 14,33 años $(D T=1,3)$.

\section{Instrumentos}

Cuestionario de Capacidades y Dificultades (Strengths and Difficulties Questionnaire, SDQ), versión autoinformada (Goodman, 1997). EI SDQ es un instrumento empleado para evaluar las dificultades conductuales y emocionales, y las capacidades prosociales en el ámbito social. El SDQ ha sido usado como un instrumento de análisis epidemiológico del estado de la salud mental en niños y adolescentes (Fonseca-Pedrero, Paino, Lemos-Girádez, y Muñiz, 2011; Ortuño-Sierra, FonsecaPedrero, Inchausti, y Sastre i Riba, 2016). Está compuesto por 25 ítems de respuesta tipo Likert de tres opciones $(0=$ No, nunca, $1=A$ veces, $2=$ Sí, siempre) que se agrupan en cinco subescalas (con cinco ítems cada una): Dificultades Emocionales, Problemas de Conducta, Dificultades de Hiperactividad, Problemas con Compañeros y Conducta Prosocial. Las primeras cuatro subescalas pueden agruparse para generar una puntuación Total de Dificultades. Cuanto mayor puntuación, mayor grado de dificultades en comportamiento y emociones, a excepción de la subescala de Conducta Prosocial, donde una puntuación más baja pertenece a un ajuste inferior en la conducta. Estudios previos indican que las puntuaciones del SDQ presentan un adecuado comportamiento psicométrico. Respecto a la fiabilidad de las puntuaciones, la versión en español ha mostrado valores de alpha apropiados que oscilan entre el .71 y el .84 (Ortuño, Chocarro, Fonseca, Sastre y Muñiz, 2015). En este estudio se utilizó la versión del SDQ validada en población española (Fonseca-Pedrero et al., 2011; OrtuñoSierra et al., 2016).

Escala Rasgo de Metaconocimiento sobre Estados Emocionales-24 (Trait Meta-Mood Scale-24, TMMS-24) (Salovey, Mayer, Goldman, Turvey y Palfai, 1995). El TMMS-24 es un autoinforme compuesto por 24 ítems y diseñado para la valoración de los aspectos reflexivos de la experiencia emocional. El formato de respuesta tipo Likert de cinco puntos $(1=$ Completamente en desacuerdo y $5=$ Completamente de acuerdo) distribuidos en tres dimensiones (ocho ítems cada una): Percepción, Comprensión y Regulación emocional. Estudios previos indican que es un instrumento de medida con un adecuado comportamiento psicométrico referido tanto a la consistencia interna (por encima de .85 para todas las subescalas y a la fiabilidad test-retest (satisfactorias variando de .60 a .83) así como a la acumulación de diferentes evidencias de validez (Fernández, Extremera y Ramos, 2004; Salguero, Fernandez-Berrocal, Balluerka y Aritzeta, 2010). En este estudio se utilizó la versión del TMMS-24 validada en población española (FernándezBerrocal, Extremera, y Ramos, 2004).

Índice de Reactividad Interpersonal (Interpersonal Reactivity Index, IRI) (Davis, 1980). El IRI es un autoinforme que consta de 28 ítems, desarrollado para evaluar la empatía desde un punto de vista multidimensional y disposicional. Consta de cuatro dimensiones de siete ítems cada una: Preocupación empática (EC; evalúa los sentimientos de afecto, compasión y preocupación hacia otros), Malestar personal (PD; mide los sentimientos de ansiedad e incomodidad resultantes al observar la experiencia negativa de otra persona), Toma de perspectiva (PT; valora los esfuerzos de adoptar la perspectiva de otras personas y ver las cosas desde su punto de vista) y Fantasía ( $F$; mide la tendencia a identificarse con los personajes de películas, novelas, obras y otras situaciones ficticias). El formato de respuesta es Likert de cinco puntos ( $1=$ No me describe nada bien; 5 = Me describe perfectamente). Para el presente estudio se utilizó la versión del IRI validada en población española (Pérez-Albéniz, De Paúl, Etxeberría, Montes y Torres, 2003), cuyas puntuaciones han mostrado una adecuada fiabilidad (entre 0,71 y 0,80 ) en muestras de estudiantes.

Escala de Autoestima de Rosenberg (Rosenberg, 1965). Este instrumento es una escala unidimensional compuesta por 10 ítems (por ejemplo, "En general estoy satisfecho conmigo mismo/a") que deben ser puntuados en una escala tipo Likert de cinco puntos ( $1=$ totalmente en desacuerdo y $4=$ totalmente de acuerdo). En el presente trabajo se ha utilizado la versión española que ha mostrado una consistencia interna mediante alpha de Cronbach de 0,87. Adicionalmente, ha evidenciado otras propiedades psicométricas adecuadas en estudios previos (Rosenberg y Owens, 2001; Vázquez, Jiménez y Vázquez, 2004).

Cuestionarios de satisfacción del programa de educación emocional. Se desarrolló un cuestionario ad hoc con dos versiones diferentes para participantes y para familias. La construcción del cuestionario siguió los estándares internacionales recomendados (Muñiz y Fonseca-Pedrero, 2019). El cuestionario 


\section{BEATRIZ LUCAS MOLINA}

de satisfacción dirigido a los participantes del estudio constó de 35 ítems. Las primeras 21 preguntas trataban sobre la satisfacción de los participantes con diferentes aspectos del programa y eran de respuesta tipo Likert de cinco puntos (1=Totalmente de acuerdo; 5=Totalmente en desacuerdo). Las diez siguientes preguntas evaluaban cada una de las sesiones en el mismo formato de respuesta que las anteriores. Se incluyó, asimismo, una pregunta sobre la valoración global del programa con respuesta tipo Likert de diez puntos (siendo el 10 la valoración más alta). Además, contenía dos preguntas de respuesta afirmativa o negativa sobre la recomendación del programa y una pregunta con respuesta abierta sobre aspectos de mejora del programa. El cuestionario para las familias de los participantes consistía en 17 ítems. Los primeros 13 ítems fueron en escala tipo Likert de cinco puntos que iban desde $1=$ Totalmente de acuerdo, a 5=Totalmente en desacuerdo, y pretendían evaluar diferentes aspectos del programa como son la duración o el impacto percibido. Asimismo, al igual que la versión para participantes, el cuestionario incluía una pregunta sobre la valoración global del programa, dos sobre la recomendación del programa, y finalizaba con una pregunta abierta sobre aspectos de mejora del programa. Este cuestionario ha sido utilizado en trabajos previos (Sigüenza-Marín, Carballido-Guisado, Pérez-Albéniz y Fonseca-Pedrero, 2019).

\section{Procedimiento}

La investigación contó con el apoyo de las Consejerías de Salud y Educación, Formación y Empleo del Gobierno de La Rioja, y se enmarcó en el Plan Regional de Salud Mental Riojano 2016-20. Se contactó con la asociación ARPANIH de La Rioja (Asociación Riojana de Padres de niños Hiperactivos). Se llevó a cabo una propuesta a todas las familias con hijos/as afectados por TDAH de entre 12 y 15 años mediante un escrito y mediante charlas informativas para facilitar a las familias la decisión de la participación de sus hijos/as en el proyecto. Los alumnos del grupo de comparación no recibieron la intervención ni de manera simultánea ni posteriormente.

Se seleccionó el programa INTEMO para ser administrado en este estudio puesto que los resultados de su implementación habían mostrado efectividad indicando que tiene efectos positivos a corto y a largo plazo en aspectos tan relevantes como el ajuste psicológico, las relaciones sociales, la empatía o las conductas disruptivas y violentas (Cabello et al., 2016; Extremera y Fernández-Berrocal, 2013; Ruíz-Aranda et al., 2013). El programa de intervención, con modificaciones respecto a su versión original, se llevó a cabo a lo largo de 10 sesiones semanales en tres grupos de ocho participantes cada uno. Dicha modificación del programa INTEMO ha sido utilizado en trabajos previos (Sigüenza-Marín et al., 2019). Las sesiones las condujeron dos miembros del equipo investigador, con grado de máster en Psicología y Educación, formados específicamente en programas de educación emocional. Ambos colaboraban y dirigían todas las sesiones conjuntamente. Los profesionales que se encargaron de la implementación del programa estaban lo suficientemente formados tanto en los modelos y programas de intervención de educación emocional como en su capacitación para poder hacer frente a los posibles problemas que surgieran durante su aplicación. Antes, durante y después de la implementación del programa, los profesionales fueron formados en los principios de la práctica basada en la evidencia para maximizar la eficacia del programa.

En todo momento se informó de la confidencialidad de las respuestas, así como del carácter voluntario de la participación.
Cabe destacar que no se facilitó ningún tipo de gratificación por la participación en el estudio. Igualmente, se solicitó la firma de un consentimiento que autorizase la participación del adolescente en la investigación. Para la elaboración del pretest y postest por parte del grupo control se contactó con cada una de las familias por teléfono y se acordaron horarios y lugares para su realización.

Análisis de los datos

En primer lugar, se calcularon los estadísticos descriptivos para las subescalas y la puntuación total de los diferentes instrumentos de medida utilizados. En segundo lugar, se compararon las puntuaciones medias en función del grupo (control y experimental) y el tiempo (pretest y postest). Para ello se llevó a cabo un Análisis Multivariado de la Varianza (MANOVA) de medidas repetidas, tomando como factor intersujetos el grupo y el tiempo como factor intrasujeto y como variables dependientes las subescalas y la puntuación total de los instrumentos de medida utilizados. Se utilizó el valor Lambda de Wilks para observar si existían diferencias significativas entre todas las variables dependientes tomadas en conjunto. Como índice de tamaño del efecto se empleó el estadístico eta cuadrado parcial ( $\eta 2$ parcial). Asimismo, se llevaron a cabo análisis post hoc para conocer las diferencias entre grupos y/o momentos temporales, se realizaron mediante pruebas t para muestras relacionadas. Finalmente, se calcularon los estadísticos descriptivos para la medida de satisfacción con el programa. Para el análisis de los datos se utilizó el programa estadístico SPSS 21.0 (IBM, 2013).

\section{Resultados}

Implementación del programa INTEMO-UR en adolescentes afectados por TDAH. El programa de intervención en educación emocional llamado INTEMO-UR para adolescentes con TDAH se implantó de manera exitosa y se extendió a lo largo de tres meses con una duración aproximada por cada sesión de 60 minutos. Al tratarse de jóvenes con un problema del comportamiento y para dedicarles una educación más individualizada, se distribuyeron al azar en tres grupos de ocho participantes cada uno. En vista del número de sesiones a realizar, así como las características de este alumnado, se adaptaron muchas de las actividades, y en ocasiones se incluyeron otras, por ello el programa fue denominado INTEMOUR para diferenciarlo de la versión INTEMO original (Extremera y Fernández-Berrocal, 2013). En la Tabla 1 se presenta de forma abreviada, las sesiones llevadas a cabo en el programa, así como el contenido y las modificaciones realizadas. 
Tabla 1. Programa INTEMO-UR. Sesiones, objetivo, contenidos y modificaciones

\begin{tabular}{llll}
\hline Sesión & Objetivo(s) & Contenidos & Modificaciones respecto al INTEMO \\
\hline 1 & & & Instrumentos de evaluación.
\end{tabular}

2

Conocer las emociones básicas y cómo estas nos ayudan.

Reconocimiento adecuado de las emociones básicas a través de imágenes y objetos.

3

Expresar emociones de diversas formas.

4

Aprender a resolver los problemas tomando decisiones adecuadas.

5

Conocer emociones útiles que permitan una mayor eficacia.

6

Reconocer y nombrar adecuadamente los sentimientos propios.

7

Adquirir un vocabulario emocional que favorezca la comunicación afectiva.

8

Desarrollar la competencia social y la toma de perspectiva.

Favorecer la actitud reflexiva.

9

Aprender a regular las emociones de los demás. Consolidar estrategias de regulación.

Expresión apropiada de las emociones de diferentes maneras.

Resolución correcta de problemas vinculados a la toma de decisiones.

Conocimiento de diversas emociones básicas según la tipología de la actividad.

Reconocimiento apropiado de los sentimientos propios.

Etiquetado correcto de las emociones.

Clasificación y conexión adecuada de palabras referidas al campo semántico de las emociones.

Respuesta más adecuada a las necesidades de los demás.

Utilización de estrategias que favorezcan la regulación emocional.
Añadir objetos en la actividad: Un viaje por el mundo de las emociones, Juegos de cartas: Ikonikus y si yo fuera.

Añadir actividad Los sombreros. Modificación de la actividad Adivina la emoción.

Receta de la felicidad.

Añadir juego Kahoot.it.

Visualización del vídeo sobre percepción.

Actividad Playlist. Dómino de emociones. Dixit. Memory. Trivial de las emociones.

Pequeñas modificaciones en el $\mathrm{Pa}$ sapalabra.

Bingo de las emociones.

Visualización de vídeos.

Scape Room.

Monopoly emociones.

Allá tú emocional.

Capítulo de la serie Merlí. Debate sobre él mismo.

Instrumentos de evaluación.

Evaluación final de los participantes. Evaluación.

Efectos del programa de educación emocional en indicadores de salud mental

En la Tabla 2 se presentan los estadísticos descriptivos para todas las puntuaciones del SDQ en los momentos temporales en función del grupo (comparación vs. intervención). Para las puntuaciones del SDQ, el valor $\lambda$ de Wilks reveló que ni el efecto del factor tiempo ( $\lambda$ Wilks $=0.992, F=0.375, p>0.544$, $\eta^{2}$ parcial $\left.=0.008\right)$ ni su interacción con el grupo $(\lambda$ Wilks $=$ $0.978, \mathrm{~F}=1.014, p=0.319, \eta^{2}$ parcial $=0.022$ ) eran estadísticamente significativos. Sin embargo, se observó una interacción estadísticamente significativa entre el tiempo, el grupo y las dimensiones del SDQ $\left(\lambda\right.$ Wilks $=0.719, \mathrm{~F}=4.195, p=0.006, \eta^{2}$ parcial $=0.281$ ).

Tal y como se observa en la Figura 1, los resultados indicaron la existencia de diferencias estadísticamente significativas en- tre los participantes del grupo de intervención entre el pretest y el postest en la dimensión de Problemas con los Compañeros $(p=0.015)$. En concreto, los participantes del grupo de intervención mostraron una puntuación media significativamente más baja después de la intervención realizada, respecto al grupo de comparación. En el resto de las dimensiones no se observaron diferencias estadísticamente significativas entre los grupos, si bien es destacable que en la dimensión Conducta Prosocial ( $p=0.074)$ se evidenció una tendencia al alza en los participantes del grupo de intervención, respecto del grupo de comparación. 
Alicia Pérez De Albéniz Iturriaga, Eduardo Fonseca Pedrero, Raquel Carballido Guisado, Víctor Santiago Sigüenza Marín,

Tabla 2. Estadísticos descriptivos del Cuestionario de Capacidades y Dificultades en los dos momentos temporales en función del grupo

\begin{tabular}{lcccc}
\hline & \multicolumn{3}{c}{ Grupo } & \multicolumn{2}{c}{$\begin{array}{c}\text { Intervención } \\
(\mathrm{n}=24)\end{array}$} \\
\hline Dimensiones & $\begin{array}{c}\text { Comparación } \\
(\mathrm{n}=24)\end{array}$ & $\begin{array}{c}\text { Pretest } \\
M(D T)\end{array}$ & $\begin{array}{c}\text { Pretest } \\
M(D T)\end{array}$ \\
\hline Problemas Emocionales & $7.87(2,3)$ & $\begin{array}{c}\text { Pretest } \\
M(D T)\end{array}$ & $8.25(2,4)$ & $7.75(2,3)$ \\
Problemas Conductuales & $8.20(2,4)$ & $8.50(3,0)$ & $8.70(2,0)$ & $8.08(2,2)$ \\
$\begin{array}{l}\text { Problemas con } \\
\text { Compañeros }\end{array}$ & $7.04(2,4)$ & $8.70(2,8)$ & $8.04(2,1)$ & $10.70(2,1)$ \\
Hiperactividad & $10.62(1,9)$ & $7.58(2,5)$ & $10.54(2,5)$ & $10.58(2,5)$ \\
Conducta Prosocial & $12.70(2,0)$ & $9.91(2,2)$ & $12.75(2,1)$ & $13.45(1,4)$ \\
\hline
\end{tabular}

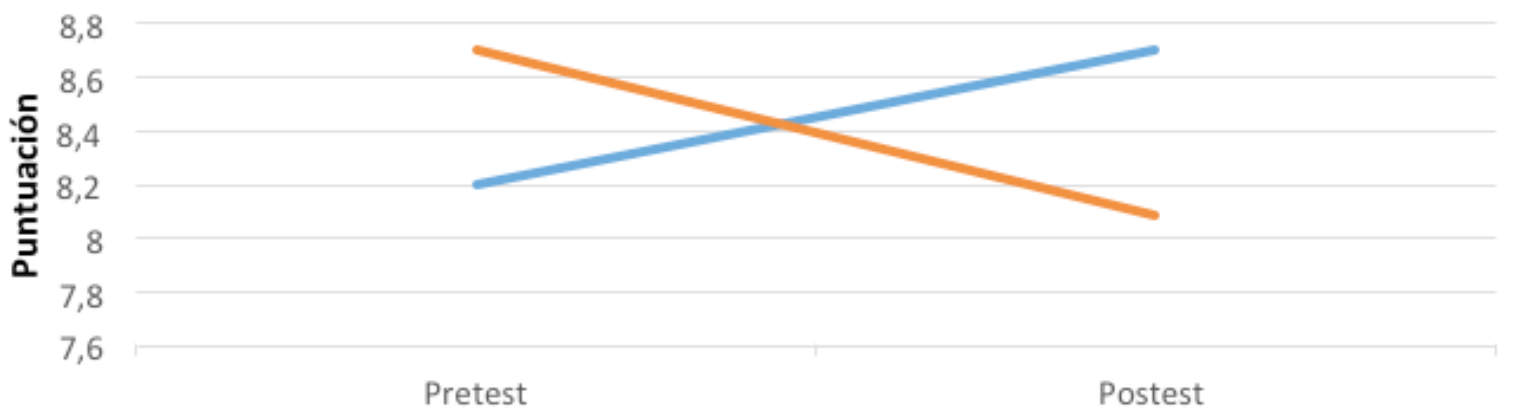

Momento temporal

Grupo comparación

Grupo intervención

Figura 1. Puntaciones medias en la dimensión Problemas conductuales (SDQ) para los grupos comparación e intervención en el pretest y postest.

Efectos del programa de educación emocional en indicadores de inteligencia emocional percibida, empatía y autoestima

En la Tabla 3 se presentan los estadísticos descriptivos para las subescalas del TMMS-24 y del IRI y la puntuación total de autoestima en los dos momentos temporales en función del grupo.

En primer lugar, en referencia a las subescalas del TMMS-24, el valor $\lambda$ de Wilks no reveló la existencia de diferencias estadísticamente significativas para el factor tiempo $(\lambda$ Wilks $=$ $0.964, F=1.713, p=.197, \eta^{2}$ parcial $\left.=0.036\right)$ ni se encontró una interacción estadísticamente significativa entre el tiempo y el grupo ( $\lambda$ Wilks $=0.968, F=1.497, p=.227, \eta^{2}$ parcial=0.032). Sin embargo, sí se observó una interacción estadísticamente significativa entre el tiempo, las subescalas del TMM24 y el grupo ( $\lambda$ Wilks $=0.806, F=5.399, p=.008, \eta^{2}$ parcial $=0.194$ ). En la Figura 2 se representa dicha interacción y se observa la existencia de diferencias estadísticamente significativas entre los participantes del grupo de intervención entre el pretest $y$ el postest en la dimensión de Regulación emocional ( $p=$
0.004). En concreto, los participantes del grupo de intervención mostraron una puntuación media significativamente más alta después de la intervención realizada, respecto al grupo de comparación. En el resto de las dimensiones no se observaron diferencias estadísticamente significativas entre los grupos. En segundo lugar, en referencia a las subescalas del IRI, el valor $\lambda$ de Wilks no reveló la existencia de diferencias estadísticamente significativas ni para el factor tiempo $(\lambda$ Wilks $=$ $0.970, F=1.445, p=.235, \eta^{2}$ parcial $\left.=0.030\right)$, ni para la interacción tiempo x grupo $(\lambda$ Wilks $=0.984, F=0.730, p=.397$, $\eta^{2}$ parcial $\left.=0.016\right)$, ni para la interacción entre el tiempo, las subescalas del IRI y el grupo ( $\lambda$ Wilks $=0.985, F=.227, p=$ $.877, \eta^{2}$ parcial $=0.015$ ).

En tercer lugar, el análisis de las diferencias en autoestima evidenció que no existían diferencias estadísticamente significativas ni en función del tiempo ( $\lambda$ Wilks $=0,998, F=0.075, p=$ $.785, \eta^{2}$ parcial $\left.=0.002\right)$, ni en la interacción del tiempo por el grupo ( $\lambda$ Wilks $=0.930, F=3.449, p=.070, \eta^{2}$ parcial $\left.=0.070\right)$. 
Tabla 3. Estadísticos descriptivos para las puntuaciones de inteligencia emocional percibida, empatía y autoestima en los dos momentos temporales en función del grupo

\begin{tabular}{|c|c|c|c|c|}
\hline & \multicolumn{4}{|c|}{ Grupo } \\
\hline & \multicolumn{2}{|c|}{$\begin{array}{l}\text { Comparación } \\
(n=24)\end{array}$} & \multicolumn{2}{|c|}{$\begin{array}{l}\text { Intervención } \\
(\mathrm{n}=24)\end{array}$} \\
\hline & $\begin{array}{l}\text { Pretest } \\
M(D T) \\
\end{array}$ & $\begin{array}{l}\text { Pretest } \\
M(D T) \\
\end{array}$ & $\begin{array}{l}\text { Pretest } \\
M(D T)\end{array}$ & $\begin{array}{l}\text { Pretest } \\
M(D T)\end{array}$ \\
\hline \multicolumn{5}{|l|}{ Inteligencia emocional } \\
\hline Percepción & $22.33(8,5)$ & $21.91(8,8)$ & $23.62(7,0)$ & $25.08(6,0)$ \\
\hline \multicolumn{5}{|l|}{ Comprensión } \\
\hline & $25.70(7,9)$ & $27.25(8,7)$ & $28.00(5,6)$ & $27.70(6,7)$ \\
\hline Regulación & $28.20(8,9)$ & $27.25(8,3)$ & $25.58(7,2)$ & $29.37(5,4)$ \\
\hline \multicolumn{5}{|l|}{ Empatía } \\
\hline Toma perspectiva & $21.29(4,9)$ & $21.75(4,6)$ & $21.87(4,1)$ & $22.41(2,7)$ \\
\hline Fantasía & $19.91(4,0)$ & $20.33(5,1)$ & $21.25(5,4)$ & $22.29(4,3)$ \\
\hline Preocupación empática & $24.75(4,5)$ & $24.70(4,5)$ & $24.95(3,9)$ & $26.45(3,8)$ \\
\hline Malestar personal & $15.29(4,8)$ & $14.95(4,9)$ & $16.00(4,0)$ & $15.87(3,7)$ \\
\hline \multicolumn{5}{|l|}{ Autoestima } \\
\hline Puntuación total & $32.70(5,5)$ & $31.25(6,0)$ & $31.87(5,2)$ & $32.95(3,5)$ \\
\hline
\end{tabular}

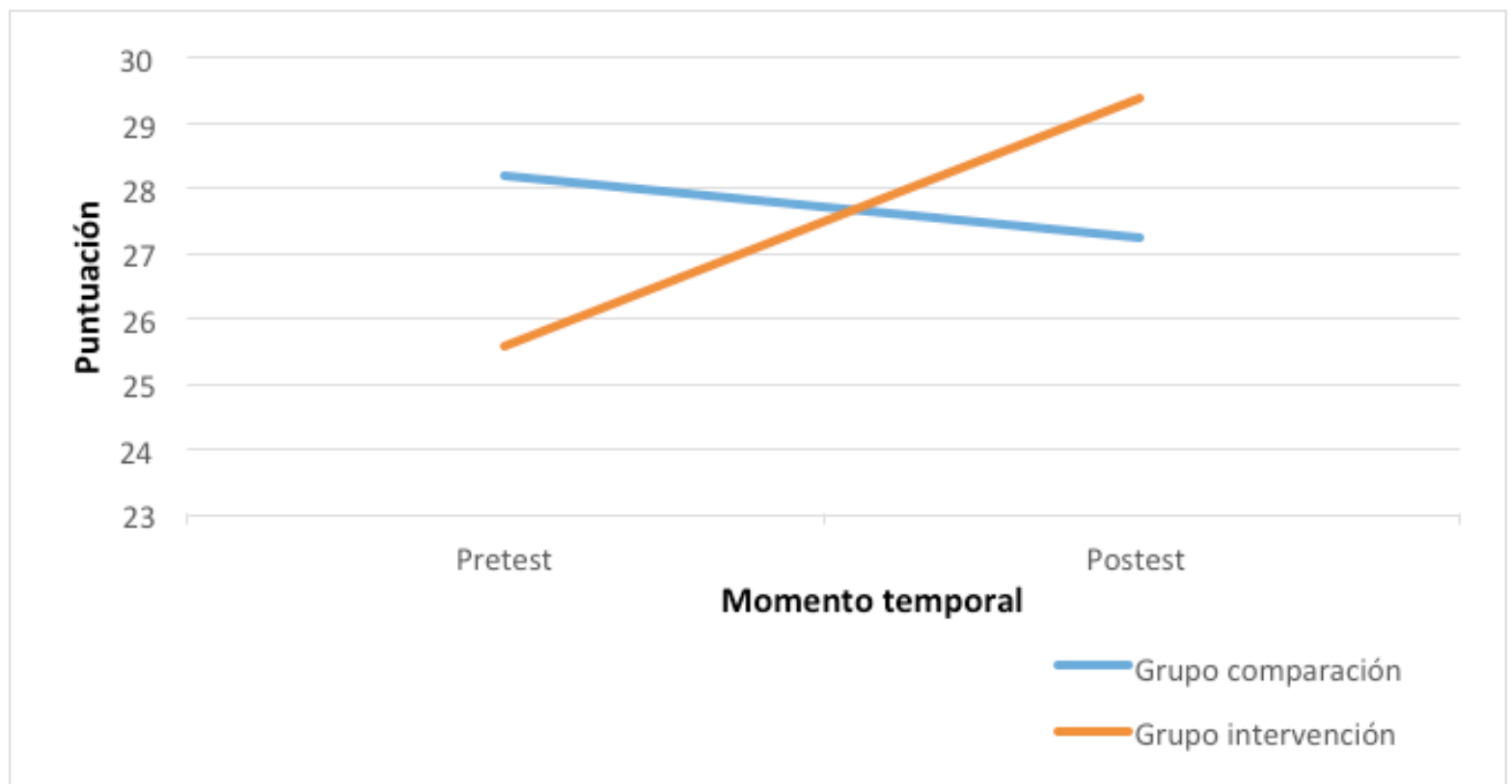

Figura 2. Puntaciones medias en la dimensión regulación emocional de inteligencia emocional (TMMS-24) para los grupos comparación y tratamiento en el pretest y postest.

Satisfacción del programa de entrenamiento de educación emocional por parte de los participantes y familiares

Los estadísticos descriptivos referidos al grado de satisfacción de los participantes con el programa de educación emocional se presentan en la Tabla 4. Los ítems que obtuvieron una mayor puntuación media fueron los que hacían referencia a la satisfacción con el profesorado y las dinámicas (siendo las mejor valoradas las sesiones 5 y 9 ).
Respecto a los ítems sobre al impacto percibido del programa (ver Tabla 5), los que obtuvieron una puntuación media más elevada fueron los que hacían referencia a la utilidad de los contenidos dados en el programa para su vida y al fomento de una mayor capacidad para comprender lo que son capaces de hacer. Asimismo, es preciso subrayar que el 100\% de los participantes afirmó que repetirían el programa y que lo recomendarían a sus amigos/as. Del mismo modo, es digno de mención que el $100 \%$ de los participantes puntuó por encima de siete puntos en la valoración general del programa, siendo la media de $9.08(D T=0.82)$. 
Por otro lado, se analizó el grado de satisfacción con el programa de las familias de los adolescentes con diagnóstico de TDAH (véase Tabla 6). Los ítems que obtuvieron una mayor puntuación media fueron los que hacían referencia a la participación de la asociación ARPANIH en la consecución de este tipo de programas, al grado de satisfacción de la participación de sus hijos/as en el programa, a la consideración de la importancia de las competencias socioemocionales en el proceso de enseñanza-aprendizaje y a la continuación del mismo en los centros educativos.
En la Tabla 7 se presentan los estadísticos descriptivos de los ítems sobre percepción del impacto del programa en los participantes según las familias. Los ítems que obtuvieron una mayor puntuación media fueron los que hacían referencia al disfrute de sus hijos/as durante el programa y a lo positivo que este había resultado para los mismos. Es preciso subrayar que el 95,8\% de las familias de los participantes afirmó que repetirían el programa y el $100 \%$ recomendarían este programa a otras familias. Finalmente, en referencia a la satisfacción general de las familias, la puntuación media fue de $8.41(D T=1.41)$.

Tabla 4. Estadísticos descriptivos de los ítems del cuestionario de satisfacción de los participantes

\begin{tabular}{|c|c|c|c|c|}
\hline & & $M(D T)$ & Asimetría & Curtosis \\
\hline \multicolumn{5}{|c|}{ Aspectos generales del programa: } \\
\hline 1. & Los contenidos han cubierto mis expectativas & $4.45(0.72)$ & -0.98 & -0.28 \\
\hline 2. & Los contenidos me han gustado & $4.08(0.71)$ & -0.89 & 2.07 \\
\hline 3. & Los temas se han tratado con la profundidad que esperaba & $4.00(0.65)$ & -0.99 & -1.16 \\
\hline 4. & Los ejercicios prácticos han sido de interés & $4.66(0.48)$ & -0.75 & -1.56 \\
\hline 5. & Las dinámicas de grupo han sido divertidas & $4.50(0.58)$ & -0.69 & -0.40 \\
\hline 6. & Me han gustado los materiales que se han dado & $4.54(0.72)$ & -1.29 & 0.34 \\
\hline 7. & El aula y mobiliario han sido adecuadas & $4.54(0.77)$ & -1.95 & 4.01 \\
\hline 8. & $\begin{array}{l}\text { Los medios y materiales utilizados para las dinámicas me han gus- } \\
\text { tado }\end{array}$ & $4.33(0.86)$ & -2.48 & 9.07 \\
\hline 9. & Me ha parecido que el programa ha durado poco tiempo & $4.87(0.33)$ & -2.42 & 4.21 \\
\hline \multicolumn{5}{|c|}{ Profesorado: } \\
\hline 10. & Los profesores dominan la materia & $4.87(0.33)$ & -2.42 & 4.21 \\
\hline 11. & Los profesores explican con claridad & $4.87(0.33)$ & -2.42 & 4.21 \\
\hline 12. & Los profesores son simpáticos & $4.91(0.28)$ & -3.22 & 9.12 \\
\hline 13. & Los profesores nos han tratado con respeto y cariño & $4.50(0.51)$ & 0.00 & -2.19 \\
\hline 14. & Los profesores motivan y despiertan mi interés & $4.20(0.83)$ & -0.91 & 0.57 \\
\hline \multicolumn{5}{|c|}{ Percepción del programa: } \\
\hline 15. & Creo que se debería impartir el programa desde el colegio/instituto & $4.58(0.50)$ & -0.36 & -2.05 \\
\hline 16. & Estoy contento con mi participación en el programa & $4.58(0.50)$ & -0.361 & -2.05 \\
\hline \multicolumn{5}{|c|}{ Sesiones: } \\
\hline 1. & Pretest & $3.04(1.16)$ & -0.63 & -0.50 \\
\hline 2. & Objetos sobre emociones & $4.33(0.70)$ & -0.57 & -0.69 \\
\hline 3. & Música emocional y el juego-sombreros & $4.54(0.58)$ & -0.87 & -0.11 \\
\hline 4. & Búnker, Kahoot y karaoke & $4.41(0.58)$ & -0.36 & -0.68 \\
\hline 5. & Dominó, Memory, Trivial y Dixit & $4.62(0.49)$ & -0.55 & -1.86 \\
\hline 6. & Pasapalabra, Bingo & $4.58(0.58)$ & -1.06 & -0.29 \\
\hline 7. & Scape Room & $4.50(0.78)$ & -1.19 & -0.15 \\
\hline 8. & Empatía & $4.33(0.76)$ & -0.70 & -0.89 \\
\hline 9. & Película & $4.66(0.70)$ & -1.89 & 2.09 \\
\hline 10. & Postest & $3.37(1.13)$ & -0.44 & -0.03 \\
\hline \multicolumn{5}{|c|}{ Valoración general: } \\
\hline 11. & Nota Final de programa & $9.08(0.82)$ & -0.66 & 0.13 \\
\hline
\end{tabular}


Tabla 5. Estadísticos descriptivos de los ítems sobre percepción del impacto del programa en los participantes

\begin{tabular}{|c|c|c|c|c|}
\hline & & $M(D T)$ & Asimetría & Curtosis \\
\hline 1. & Los contenidos aprendidos han sido útiles para mi vida & $4.29(0.80)$ & -0.60 & -1.17 \\
\hline 2. & Creo que el programa ha mejorado mis recursos emocionales & $4.12(0.85)$ & -0.71 & 0.03 \\
\hline 3. & El programa me ha ayudado a mejorar mis relaciones con los demás & $4.04(1.04)$ & -0.84 & -0.36 \\
\hline 4. & Desde que vengo al programa conozco mejor lo que soy capaz de hacer & $4.33(0.70)$ & -1.40 & 3.96 \\
\hline
\end{tabular}

Tabla 6. Estadísticos descriptivos de los ítems del cuestionario de satisfacción de las familias de los participantes

\begin{tabular}{|c|c|c|c|c|}
\hline & & $M(D T)$ & Asimetría & Curtosis \\
\hline \multicolumn{5}{|c|}{ Aspectos generales del programa: } \\
\hline 1. & $\begin{array}{l}\text { Valoro positivamente que ARPANIH colabore con la Universidad de La Rioja y la } \\
\text { Consejería en la consecución de este tipo de actividades para los adolescentes }\end{array}$ & $4.95(0.20)$ & -4.89 & 4.00 \\
\hline 2. & $\begin{array}{l}\text { Considero que los adolescentes con una mayor Inteligencia Emocional son tam- } \\
\text { bién los que obtienen las mayores calificaciones académicas }\end{array}$ & $3.79(1.31)$ & -0.57 & -1.05 \\
\hline 3. & $\begin{array}{l}\text { Considero que los alumnos/as que obtienen mayor Inteligencia Emocional son } \\
\text { más propensos a comportamientos prosociales }\end{array}$ & $4.37(0.71)$ & -0.70 & -0.62 \\
\hline 4. & $\begin{array}{l}\text { Como padre/ madre considero que la Inteligencia Emocional es esencial en el } \\
\text { proceso de Enseñanza-Aprendizaje. }\end{array}$ & $4.66(0.56)$ & -1.52 & 1.62 \\
\hline \multicolumn{5}{|c|}{ Percepción del programa: } \\
\hline 5. & Se debería continuar impartiendo este programa en el colegio/instituto. & $4.66(0.56)$ & -1.52 & 1.62 \\
\hline 6. & Estoy contento con la participación de mi hijo/a en el programa & $4.75(0.53)$ & -2.13 & 4.14 \\
\hline \multicolumn{5}{|c|}{ Valoración general: } \\
\hline 7. & Nota Final de programa & $8.41(1.41)$ & -0.72 & 0.16 \\
\hline
\end{tabular}

Tabla 7. Estadísticos descriptivos de los ítems sobre percepción del impacto del programa en los participantes según las familias

\begin{tabular}{|c|c|c|c|c|}
\hline & & $M(D T)$ & Asimetría & Curtosis \\
\hline 1. & El programa ha mejorado la capacidad de gestión de las emociones de mi hijo/a & $3.45(0.65)$ & 1.16 & 0.35 \\
\hline 2. & El programa ha mejorado el rendimiento académico de mi hijo/a & $3.04(0.75)$ & 1.27 & 2.78 \\
\hline 3. & El programa ha mejorado el clima familiar & $3.37(0.76)$ & 0.45 & 0.17 \\
\hline 4. & El programa ha ayudado a mejorar las relaciones interpersonales de mi hijo/a & $3.50(0.83)$ & 0.73 & -0.34 \\
\hline 5. & Mi hijo/a he mejorado sus competencias socioemocionales & $3.50(0.65)$ & 0.99 & 0.00 \\
\hline 6. & Creo que mi hijo/a ha disfrutado de las actividades realizadas & $4.62(0.57)$ & -1.28 & 0.85 \\
\hline 7. & El programa ha sido positivo para mi hijo/a & $4.58(0.65)$ & -1.35 & 0.81 \\
\hline
\end{tabular}

\section{Discusión}

El principal objetivo de este trabajo fue analizar la eficacia de un programa de educación emocional en adolescentes con diagnóstico de TDAH con el propósito de mejorar sus competencias socioemocionales. Los resultados parecen indicar que el programa INTEMO (con modificaciones) fue adaptado de manera exitosa a este contexto de investigación. La utilización de un grupo de intervención y uno de control, con diagnóstico de TDAH evaluado en paralelo, permitió analizar la eficacia del programa mediante la utilización de diferentes indicadores psicométricos de salud mental, inteligencia emocional, empatía y autoestima. Asimismo, los usuarios del programa mostraron un alto nivel de satisfacción y adherencia hacia el mismo. En primer lugar, los resultados evidenciaron la existencia de mejoras en los participantes del grupo de intervención, respecto al grupo de comparación, tras la intervención en indica- dores de salud mental e inteligencia emocional percibida. En concreto se observó una disminución en problemas con los compañeros y una mejoría (sin ser estadísticamente significativa) en conducta prosocial. Estos resultados están en consonancia con estudios previos (p. ej., Miranda, Presentación, y Soriano, 2002; Shelton et al., 2000; Sibley et al., 2011, 2013) que mostraron mejoras en relación con problemas sociales de adolescentes que presentaban la misma problemática. Sin embargo, difieren de estudios como los de Hoza et al. (2005) que afirmaron no encontrar mejoras en las relaciones entre los compañeros. En referencia a los problemas conductuales, los resultados mostraron una mejora que no llegó a ser estadísticamente significativa, como se había observado en estudios previos (Miranda et al., 2002; Shelton et al., 2000; Sibley et al., 2011, 2013). Al mismo tiempo, tras la administración del programa se encontraron diferencias estadísticamente significativas en inteligencia emocional percibida, concretamente 


\section{BEATRIZ LUCAS MOLINA}

en el componente de regulación emocional. Estos resultados son similares a los hallados en estudios previos como el de Nelis y colaboradores (2009) con adolescentes de la población general o como los de García (2012) y Montañez (2014), cuya intervención se realizó en jóvenes con TDAH. Por último, tras la intervención, no se encontraron mejorías en los niveles de empatía o autoestima informados por los participantes del grupo de intervención.

En cualquier caso, las mejorías encontradas en indicadores de salud mental y en regulación emocional tras la intervención de un programa de educación emocional en alumnado con diagnóstico de TDAH, son cuanto menos esperanzadoras. Además, estos resultados hallados permiten tomar decisiones informadas en función de la evidencia empírica. Los adolescentes con diagnóstico de TDAH son descritos frecuentemente en la literatura científica como impulsivos, problemáticos a nivel conductual y con dificultades en las relaciones sociales (p. ej., Barkley, 2014; Fernández-Jaén et al., 2011; Wehmeier, Schacht y Barkley, 2010), por lo que los programas que tratan de desarrollar, fomentar y/o potenciar las competencias socioemocionales son una estrategia de intervención necesaria. Además, no se debe perder de vista que la investigación muestra la importancia de intervenir en edades tempranas para que las dificultades no se cronifiquen posteriormente en la edad adulta (Fernández-Berrocal, Cabello y GutiérrezCobo, 2017). En general, los programas de aprendizaje social y emocional parecen aumentar de forma efectiva la competencia social, la competencia emocional, la autorregulación conductual y los resultados del aprendizaje, así como reducir las dificultades conductuales y emocionales en los niños y adolescentes (p.ej., Blewitt et al., 2018; Taylor et al., 2017). Este tipo de programas no solo tienen impacto en el propio alumnado, sino que afectan también a su entorno familiar, social, escolar y sanitarios, por lo que los beneficios son a múltiples niveles. Atender estas necesidades mediante intervenciones basadas en la evidencia, como la descrita en el presente estudio, que muestran mejorías (tras 3 meses) en aspectos tan centrales para la vida de los jóvenes con diagnóstico de TDAH, debe alentar, sin lugar a duda, el trabajo futuro en este ámbito.

En este estudio también se analizó el grado de aceptabilidad del programa por parte de los usuarios mediante el análisis de la satisfacción de los alumnos y sus familiares al mismo. Los resultados indicaron que todos los participantes de la intervención recomendarían el programa, y volverían a repetirlo. Igualmente, las familias de los usuarios reflejaron que un $95,8 \%$ repetiría este curso y el 100\% recomendarían este programa a otras familias. En vista de estos indicadores tan positivos, se puede afirmar que tanto participantes como familiares presentaban una alta satisfacción en general con el programa implementado. Estos resultados son totalmente convergentes con los encontrados en trabajos previos llevados a cabo en adolescentes de la población general (Sigüenza-Marín et al., 2019).

Referente a los resultados encontrados en el presente trabajo, son dignos de mención los siguientes comentarios. En primer lugar, los resultados parecen indicar que el programa de educación emocional ha mejorado algunos componentes socioemocionales de los participantes, como la regulación emocional percibida. Cierto es que no se han encontrado diferencias entre grupos en autoestima o empatía, si bien estos dos componentes no son un ingrediente esencial en las competencias que se trabajan en programa de intervención. En este sentido, trabajos previos utilizando el INTEMO y diferentes programas de aprendizaje socioemocional (Social-Emotional Learning) han demostrado su efectividad y utilidad, indicando efectos positivos a corto, medio y largo plazo en aspectos tan relevantes como el ajuste psicológico, las relaciones sociales, la empatía, las conductas disruptivas o el rendimiento académico (p.ej., Blewitt et al., 2018; Cabello et al., 2016; Castillo et al., 2013; Fernández-Martínez, Morales, Espada, Essau, y Orgilés, 2019; Taylor et al., 2017).

En segundo lugar, es posible que los instrumentos de medida utilizados no hayan permitido captar las diferencias entre los grupos. Por ejemplo, es plausible que las herramientas de medición no sean sensibles al cambio o que el constructo que pretenden medir sea más bien de naturaleza rasgo y no estado. Digno de mención es que los instrumentos utilizados para medir la efectividad del programa fueron autoinformes que, aunque tienen aspectos positivos (p.ej., facilidad de administración en muestras amplias de la población general), también tienen limitaciones (p.ej., capacidad de introspección, deseabilidad social, sesgos de respuesta).

En tercer lugar, el tamaño muestral empleado en este trabajo fue reducido. Además, el muestreo utilizado fue de tipo incidental. Estos dos aspectos, entre otros, son limitaciones potenciales que pueden afectar a la obtención de resultados estadísticamente significativos y a la validez externa.

En cuarto lugar, en este trabajo no se ha realizado un seguimiento a medio o largo plazo de los participantes, aspecto que hubiese sido interesante de cara a examinar si emergen o no nuevas diferencias o si aumenta o no el tamaño del efecto entre los grupos con el paso del tiempo, por ejemplo, a los 6 o 12 meses.

En quinto lugar, se ha utilizado un tipo de análisis estadístico dentro del modelo lineal general (MANOVA) que compara puntuaciones medias de grupos de participantes. Aunque haya ausencia de resultados estadísticamente significativos a nivel de grupo en algunas variables (p.ej., autoestima), es plausible que a nivel intraindividual existan cambios. Por ejemplo, es posible que algunos de los adolescentes del grupo de intervención hayan experimentado mejoría en determinadas competencias socioemocionales. Sería interesante en futuros estudios examinar las diferencias intraindividuales, así como la estabilidad temporal de las puntaciones.

Finalmente, más allá de la significación estadística, también hay vida. Como investigadores, y considerando, por ejemplo, las puntuaciones de satisfacción al programa referidas por los usuarios (indicador de la aceptabilidad del programa), la adherencia al programa (en función del número de sesiones a las que han acudido) o el comportamiento observado por los investigadores en las sesiones de intervención, estamos plenamente convencidos de la utilidad de este tipo de programas en este campo de actuación. El beneficio de estos programas no debe medirse solo en función de la significación estadística ( $p$-valor), sino también en su impacto personal y social.

En suma, los resultados indicaron que la adaptación e implementación del programa fue exitosa, mostró su eficacia y los usuarios afirmaron sentirse satisfechos con la intervención. Estos hallazgos indican la conveniencia de continuar con el desarrollo y potenciación de las competencias socioemocionales en esta etapa del desarrollo con el propósito de mejorar 
el bienestar personal, familiar, académico y social de los más jóvenes, tengan o no un problema emocional o comportamental. No se debe perder de vista que los jóvenes son el principal capital de nuestra sociedad presente y futura.

\section{Referencias}

American Psychiatric Association (2014). Manual Diagnóstico y Estadístico de los Trastornos Mentales-5 (DSM-5). Madrid: Editorial Médica Panamericana.

Barkley, R. (2014). Attention-Deficit Hyperactivity Disorder Fourth Edition. A Handbook for Diagnosis and Treatment. UK: The Guilford Press.

Blewitt, C., Fuller-Tyszkiewicz, M., Nolan, A., Bergmeier, $\mathrm{H}_{\text {., }}$ Vicary, D., Huang, T., McCabe, P., McKay, T., y Skouteris, H. (2018). Social and emotional learning associated with universal curriculum-based interventions in early childhood education and care centers: a systematic review and meta-analysis. JAMA Netw Open, 1(8):e185727. doi: 10.1001/jamanetworkopen.2018.5727

Bisquerra, R. (2011). Educación emocional: propuesta para educadores y familiares. Bilbao: Desclée de Brouwer.

Cabello, R., Castillo, R., Rueda, P., y Fernández-Berrocal, P. (2016). Programa INTEMO+. Mejorar la inteligencia emocional de los adolescentes. Madrid: Pirámide.

Campeño-Martínez, Y., Gázquez-Linares, J. , y Santiuste-Bermejo, V. (2016). Medida de las emociones en niños con Trastorno por Déficit de Atención con Hiperactividad (TDAH). Un ensayo experimental con la técnica de potenciales evocados. Universitas Psychologica, 15(5). doi: 10.11144/Javeriana.upsy15-5. ment

Castillo, R., Salguero, J., Fernández-Berrocal, P., y Balluerka, N. (2013). Effects of an emotional intelligence intervention on aggression and empathy among adolescents. Journal of Adolescence, 36(5), 883-892.

Davis, M.H. (1980). A multidimensional approach to individual differences in empathy. Catalog of Selected Documents in Psychology, 10, 85, 1-17.

Decety, J., y Svetlova, M. (2012). Putting together phylogenetic and ontogenetic perspectiveson empathy. Developmental Cognitive Neuroscience, 2 (1), 1-24.

Evans, S., Axelrod, J., y Langberg, J. (2004). Efficacy of a schoolbased treatment program for middle school youth with ADHD: pilot data. Behavior Modification, 28(4), 528-547.

Evans, S., Langberg, J., Egan, T., y Molitor, S. (2014). Middle school-based and high school-based interventions for adolescents with ADHD. Child and Adolescent Psychiatry Clinick of North America, 23, 699-715.

Evans, S., Schultz, B., Demars, C., y Davis, H. (2011). Effectiveness of the challenging horizons after-school program for young adolescents with ADHD. Behavior Therapy, 42(3), 462-474.

Extremera, N., y Fernández-Berrocal, P. (2013). Inteligencia emocional en adolescentes. Padres y Maestros, 13, 34-39.

Fernández-Martínez, I., Morales, A., Espada, J., Essau, C., y Orgilés, M. (2019). Effectiveness of the program Super Skills For Life in reducing symptoms of anxiety and depression in young Spanish children. Psicothema, 31, 298-304.

Fernández-Berrocal, P., Extremera, N., y Ramos, N. (2004).
Validity and reliability of the spanish modified version of the Trait Meta-Mood Scale. Psychological Reports, 94, 751-755.

Fernández-Berrocal, P., Cabello, R., y Gutiérrez-Cobo, M. (2017). Avances en la investigación sobre competencias emocionales en educación. Revista Interuniversitaria de Formación del Profesorado, 88, 15-26.

Fernández-Berrocal, P., Extremera, N., y Ramos, N. (2004). Validity and reliability of the Spanish modified version of the Trait Meta-Mood Scale. Psychological Reports, 94, 751-755.

Fernández-Jaén, $A_{1}$, Fernández-Mayoralas, D., López-Arribas, S., García-Savaté, C., Muñiz-Borrega, B., PardosVéglia, A., Muñoz-Jareño, N. (2011). Trastorno por déficit de atención/hiperactividad y su relación con las habilidades sociales y de liderazgo evaluadas a través de un sistema de evaluación de la conducta de niños y adolescentes (BASC). Actas Españolas de Psiquiatría, 39, 339-348.

Fonseca-Pedrero, E. (2018). Análisis de redes en psicología. Papeles del Psicólogo, 39, 1-12.

Fonseca-Pedrero, E., Paino, M., Lemos-Girádez, S., y Muñiz, J. (2011). Prevalencia de la sintomatología emocional y comportamental en adolescentes españoles a través del Strengths and Difficulties Questionnaire (SDQ). Revista de Psicopatología y Psicología Clínica, 16, 15-25.

Garaigordobil, M., Cruz, S., y Pérez, J. (2003). Análisis correlacional y predictivo del autoconcepto con otros factores conductuales, cognitivos y emocionales de la personalidad durante la adolescencia. Estudios de Psicología, 24 (1), 113-134.

García de Vinuesa, González Pardo y Pérez Álvarez, M. (2014). Volviendo a la normalidad: la invención del TDAH y el trastorno bipolar infantil. Madrid: Alianza.

González, C., Navarro, I., Garu, S., Galipienso, A., y Fernández, F. (2014). Estrategias de optimización de alumnado con Trastorno por Déficit de Atención e Hiperactividad TDA-H. INFAD Revista de Psicología, 7 (1), 85-94.

Goodman, R. (1997). The Strengths and Difficuties Questionnaire: A research note. Journal of Child Psychology and Psychiatry, 38, 581-586.

IBM Corp Released. IBM SPSS Statistics for Windows, Version 22.0. Armonk, NY: IBM Corp; 2013.

Larson, R, Moneta, G., Richards, M. y Wilson, S. (2001). Continuity, stability, and change in daily emotional experience across adolescence. Child Development, 73, 1151-1165.

López, C., Sánchez, A., Pérez-Nieto, M., y Fernández, M. (2008). Impulsividad, autoestima y control cognitivo en la agresividad del adolescente. EduPsykhé, 7 (1), 81-99

Mayer, J. y Salovey, P. (1997). What is emotional intelligence? En P. Salovey y D. Sluyter (Eds.), Emotional Development and Emotional Intelligence: Implications for Educators (pp. 3-31). New York: Basic Books.

Mena, B., Nicolau, R., Salat, L., Tort, P., y Romero, B. (2008). El alumno con TDAH. Trastorno por Déficit de Atención con o sin Hiperactividad. Madrid: Ediciones Mayo.

Miranda, A., Presentación, M., y Soriano, M. (2002). Effectiveness of a school-based multicomponent program 
for the treatment of children with ADHD. Journal of Learning Disabilities, 35, 546-562.

Muñiz, J. y Fonseca-Pedrero, E. (2019). Diez pasos para la construcción de un test. Psicothema, 31, 7-16.

Nelis, D., Quoidbach, J., Mikolajczak, M., y Hansenne, M. (2009). Increasing emotional intelligence: (How) is it possible? Personality and Individual Differences, 47, 36-41.

Ortuño-Sierra, J., Chocarro, E., Fonseca-Pedrero, E., Sastre i Riba, S., y Muñiz, J. (2015). The assessment of emotional and behavioural problems: Internal structure of The Strengths and Difficulties Questionnaire. International Journal of Clinical and Health Psychology, 15, 265-273.

Ortuño-Sierra, J., Fonseca-Pedrero, E., Inchausti, F., y Sastre i Riba, S. (2016). Evaluación de dificultades emocionales y comportamentales en población infantojuvenil: El cuestionario de capacidades y dificultades (SDQ). Papeles del Psicólogo, 37(1), 14-26.

Padrós, F., Zamora, M., Salinas, P., y Martínez, M. (2016). Rasgos del trastorno por déficit de atención e hiperactividad y consumo patológico de alcohol en adolescentes. Revista Acta Psiquiátrica y Psicológica de América Latina., 62(3), 164-170.

Pérez-Álvarez, M. (2018). Para pensar la psicología más allá de la mente y el cerebro: un enfoque transteórico. Papeles del Psicólogo, 39, 161-173.

Rosenberg, M. (1965). Society and the adolescent self image. Princeton: Princeton University Press.

Rosenberg, M., y Owens, T. (2001). Low self-esteem people: A collective portrait. En T. J. Owens, S. Stryker y N. Goodman (Eds.), Exending self este-em theory and research: Sociological and psychological currents. New York, NY: Cambridge University Press.

Ruiz-Aranda, D., Cabello, R., Salguero, J., Palomera, R., Extremera, N., y Fernández-Berrocal, P. (2013). Guía para mejorar la inteligencia emocional de los adolescentes. Programa INTEMO. Madrid: Pirámide.

Salguero, J., Fernandez-Berrocal, F., Balluerka, N., y Aritzeta, A. (2010). Measuring perceived emotional intelligence in the adolescent population: psychometric properties of the trait meta-mood scale. Social Behavior and Personality, 38, 1197-1210.

Salovey, P. (1997). "What is emotional intelligence?" En P. Salovey y D. Sluyter (Eds), Emotional Development and Emotional Intelligence: Implication for Educators (pp. 3-31). New York: Basic Books.

Salovey, P., Mayer, J., Goldman, S., Turvey, C., Palfai, T. (1995). Emotional attention, clarity, and repair: exploring emotional intelligence using the Trait Meta-Mood Scale. En J. W. Pennebaker (Ed.), Emotion, Disclosure, y Health (pp. 125-151). Washington: American Psychological Association.

Sasser, T., Kalvin, C., y Bierman, K. (2016). Developmental trajectories of clinically significant attention-deficit/ hyperactivity disorder (ADHD) symptoms from grade 3 through 12 in a high-risk sample: Predictors and outcomes. Journal of Abnormal Psychology, 125, 207-219.

Shelton, T., Barkley, R., Crosswait, C., Moorehouse, M., Fletcher, K., Barrett, L., Metevia, L. (2000). Multimethod psychoeducational intervention for preschool children with disruptive behavior: two-year post-treatment follow-up. Journal of Abnormal Child Psychology,
28(3), 253- 266.

Sibley, M., Pelham, W., Derefinko, K., Kuriyan, A., Sánchez, F., y Graziano, P. (2013). A Pilot Trial of Supporting teens' Academic Needs Daily (STAND): A ParentAdolescent Collaborative Intervention for ADHD. Journal of Psychopathology and Behavioral Assessment, 35(4), 436-449.

Sibley, M., Pelham, W., Evans, S., Gnag y, E., Ross, J., y Greiner, A. (2011). An Evaluation of a summer treatment program for adolescents with ADHD. Cognitive and Behavioral Practice, 18(4), 530-544.

Sigüenza-Marín, V., Carballido-Guisado, R., Pérez-Albéniz, A., y Fonseca-Pedrero, E. (2019). Implementación y evaluación de un programa de inteligencia emocional en adolescentes. Universitas Psychologica, $18,1-13$.

Storebø, O., Elmose Andersen, M., Skoog, M., Joost Hansen, S., Simonsen, E., Pedersen, N., Tendal, B., Callesen, H., Faltinsen, E., y Gluud, C. (2019). Social skills training for attention deficit hyperactivity disorder (ADHD) in children aged 5 to 18 years. Cochrane Database Systematic Review, 21, CD008223.

Taylor, R., Oberle, E., Durlak, J., y Weissberg, R. (2017). Promoting positive youth development through school-based social and emotional learning interventions:A meta-analysis of follow-up effects. Child Development, 88, 1156-1171.

Vázquez A., Jiménez R., y Vázquez-Morejón R. (2004). Escala de autoestima de Rosenberg: fiabilidad y validez en población clínica española. Apuntes de Psicología, 22, 247-55.

Wehmeier, P., Schacht, A., y Barkley, R. (2010). Social and emotional impairment in children and adolescents with ADHD and the impact on quality of life. Journal of Adolescent Health, 46, 209-217.

Willcutt, E. (2012). The prevalence of DSM-IV Attention-Deficit/Hyperactivity Disorder: A meta-analytic review. Neurotherapeutics, 9(3), 490-499. 